\title{
Phosphoproteomics-Based Profiling of Kinase Activities in Cancer Cells
}

\author{
Jakob Wirbel, Pedro Cutillas, and Julio Saez-Rodriguez
}

\begin{abstract}
Cellular signaling, predominantly mediated by phosphorylation through protein kinases, is found to be deregulated in most cancers. Accordingly, protein kinases have been subject to intense investigations in cancer research, to understand their role in oncogenesis and to discover new therapeutic targets. Despite great advances, an understanding of kinase dysfunction in cancer is far from complete.

A powerful tool to investigate phosphorylation is mass-spectrometry (MS)-based phosphoproteomics, which enables the identification of thousands of phosphorylated peptides in a single experiment. Since every phosphorylation event results from the activity of a protein kinase, high-coverage phosphoproteomics data should indirectly contain comprehensive information about the activity of protein kinases.

In this chapter, we discuss the use of computational methods to predict kinase activity scores from MS-based phosphoproteomics data. We start with a short explanation of the fundamental features of the phosphoproteomics data acquisition process from the perspective of the computational analysis. Next, we briefly review the existing databases with experimentally verified kinase-substrate relationships and present a set of bioinformatic tools to discover novel kinase targets. We then introduce different methods to infer kinase activities from phosphoproteomics data and these kinase-substrate relationships. We illustrate their application with a detailed protocol of one of the methods, KSEA (Kinase Substrate Enrichment Analysis). This method is implemented in Python within the framework of the open-source Kinase Activity Toolbox (kinact), which is freely available at http://github.com/saezlab/kinact/.
\end{abstract}

Key words Phosphoproteomics, Mass-spectrometry, Kinase activity, Computational biology, Cancer systems biology, Signal transduction

\section{Introduction}

Protein kinases are major effectors of cellular signaling, in the context of which they form a highly complex and tightly regulated network that can sense and integrate a multitude of external stimuli or internal cues. This kinase network exerts control over cellular processes of fundamental importance, such as the decision between proliferation and apoptosis [1]. Deregulation of kinase signaling can lead to severe diseases and is observed in almost every type of cancer [2]. For instance, a single constitutively active kinase, 
originating from the fusion of the $B C R$ and $A B L$ genes, can give rise to and sustain chronic myeloid leukemia [3]. Accordingly, the small molecule inhibitor of the BCR-ABL kinase, Imatinib, has shown unprecedented therapeutic effectiveness in affected patients [4].

Fueled by these promising clinical results, due to the essential role for kinases in the patho-mechanism of cancer, and because kinases are in general pharmacologically tractable [5], a range of new kinase inhibitors has been approved or is in development for different cancer types [6]. However, not all eligible patients respond equally well, and in addition, cancers often develop resistance to initially successful therapies. This calls for a deeper understanding of kinase signaling and opens up the possibility of exploiting this knowledge therapeutically [7].

By definition, the activity of a kinase is reflected in the occurrence of phosphorylation events catalyzed by this kinase. Thus, analysis of kinase activity was traditionally achieved by monitoring the phosphorylation status of a limited number of sites known to be targeted by the kinase of interest using immunochemical techniques [8]. This, however, requires substantial prior-knowledge and yields a comparably low throughput. Other approaches exist, e.g., protein kinase activity assays $[9,10]$ or attempts to measure kinase activity with chromatographic beads functionalized with ATP or small molecule inhibitors [11].

Mass spectrometry-based techniques to measure phosphorylation can identify thousands of phosphopeptides in a single sample with ever-increasing coverage, throughput, and quality, nourished by technological advances and dramatically increased performance of MS instruments in recent years [12-14]. High-coverage phosphoproteomics data should indirectly contain information about the activity of many active kinases. The high-content nature of phosphoproteomics data, however, poses challenges for computational analysis. For example, only a small subset of the described phosphorylation sites can be explicitly associated with functional impact [15].

As a means to extract functional insight, methods to infer kinase activities from phosphoproteomics data based on priorknowledge about kinase-substrate relationships have been put forward [16-19]. The knowledge about kinase-substrate relationships, compiled in databases like PhosphoSitePlus [20] or Phospho.ELM [21], covers only a limited set of interactions. Alternatively, computational resources to predict kinase-substrate relationships based on kinase recognition motifs and contextual information have been used to enrich the collections of substrates per kinase $[22,23]$, but the accuracy of such kinase-substrate relationships has not been validated experimentally for most cases. The inferred kinase activities can in turn be used to reconstruct 
kinase network circuitry or to predict therapeutically relevant features such as sensitivity to kinase inhibitor drugs [17].

In this chapter, we start with a brief description of phosphoproteomics data acquisition, highlighting challenges for the computational analysis that may arise out of the experimental process. Subsequently, we will present different computational methods for the estimation of kinase activities based on phosphoproteomics data, preceded by the kinase-substrate resources these methods use. One of these methods, namely KSEA (Kinase-Substrate Enrichment Analysis), will be explained in more detail in the form of a guided, stepwise protocol, which is available as part of the Python opensource Toolbox kinact (for Kinase Activity Scoring) at http://www. github.com/saezlab/kinact/.

\section{Phosphoproteomics Data Acquisition}

For a summary of technical variations or available systems for the experimental setup of phosphoproteomics data acquisition, we would like to refer the interested reader to dedicated publications such as $[24,25]$. We provide here a short overview about the experimental process to facilitate the understanding of common challenges that may arise for the data analysis that we will focus on.

Mass spectrometry-based detection of peptides with posttranslational modifications (PTM) usually requires the same steps, independent of the modification of interest: (1) cell lysis and protein extraction with special focus on PTM preservation, (2) digestion of proteins with an appropriate protease, (3) enrichment of peptides bearing the modification of interest, and (4) analysis of the peptides by LC-MS/MS [26]. After the experimental work, additional data processing steps are required to identify the position of the modification, e.g., the residue that is phosphorylated. For almost every step, different protocols are available, starting from various proteases for protein digestion to different data acquisition methods for MS [24].

2.1 Phosphopeptide Enrichment
Naturally, the enrichment of phosphopeptides is a pivotal step for phosphoproteomics. Next to the enrichment method used, the choice of the protease [27] or the MS ionization method [28] also has an impact on the part of the phosphoproteome that is sampled. For phosphopeptide enrichment, the field is dominated by immobilized metal affinity chromatography (IMAC) and metal oxide affinity chromatography (MOAC), which all exploit the affinity of the phosphorylation toward metal ions. Popular techniques include $\mathrm{Fe}^{3+}$ IMAC, $\mathrm{Ti}^{4+}$-IMAC [29], or $\mathrm{TiO}_{2}$-MOAC. Alternatively, more traditional biochemical methods involving immunoaffinity purification are also in use for enrichment of phosphopeptides, although these are generally limited to studies of phosphotyrosine [30]. 
2.2 Data Acquisition
Of note, the different enrichment methods show little overlap in the detected phosphopeptides, although this can also be observed for replicates of runs using the identical enrichment method, as discussed below [31].

After enrichment, the phosphopeptides are separated chromatographically, usually by reversed phase liquid chromatography (RPLC), and then enter the mass spectrometer for tandem MS analysis (MS/MS), completing the workflow of LC-MS/MS. Variations in the chromatography method used as well as the multitude of mass spectrometry instrument types are reviewed in detail elsewhere [24]. Generally, the quality of the chromatographic separation will have a big impact on the number of phosphopeptides that can confidently be identified. Chromatography runs of higher quality also take more time, so that a tradeoff between resolution and throughput must be devised for each experiment.

For most phosphoproteomics studies so far, the MS instrument is operated in the data-dependent acquisition (DDA) mode. Therein, precursor ions from a first survey scan are selected-typically based on relative ion abundance-in order to generate fragmentation spectra in a second MS run [32], for which a database search yields the corresponding peptide sequences [33]. As a result, peptide detection in DDA is on the one hand biased toward high abundance species, but also considerably irreproducible due to stochastic precursor ion selection [34]. This inherent under-sampling of DDA usually leads to missing data points in LC-MS/MS datasets. However, this problem may be solved to some extent by extracting ion chromatograms of the peptides that are missing in some of the runs that are being compared [35-38], by matching across samples [39], or with the accurate mass and retention tag method [40].

In an alternative operation mode, selected reaction monitoring/multiple reaction monitoring (SRM/MRM), the presence and abundance of only a limited set of pre-specified peptides with known fragmentation spectra is surveyed [41]. This targeted approach overcomes many of the issues of shotgun methods, but is usually not feasible for large-scale investigation of the complete phosphoproteome.

Data-independent acquisition (DIA), e.g., SWATH-MS [42] tries to address the shortcoming of both established data acquisition strategies in order to combine the throughput of DDA with the reproducibility of SRM. In DIA, fragmentation spectra are generated for all precursor ions in a specific window of $m / z$ ratios, leading to a complete map of fragmentation spectra, followed by computational extraction of quantitative information for known spectra. For phosphoproteomics, DIA-MS has already been applied to investigate insulin signaling [43] or histone modifications [44]. However, the spectra generated by DIA-MS are usually highly complex and require intricate data extraction techniques, 
which is even more challenging for modified peptides. Recently, a computational resource for the detection of modified peptides has been put forward [45]. Overall, the available methods for DIA have as yet to mature in order to challenge the use of DDA in large-scale studies of the phosphoproteome [24].

\subsection{Quantitative} Phosphoproteomics
As for regular proteomics, several experimental methods or postacquisition tools exist to quantitate detected phosphopeptides. Those can roughly be divided into isotope labeling and label-free quantitation. In general, stable isotope labeling requires more experimental effort than label-free quantitation, but at the same time enables multiplexing of samples with different isotopes or combinations.

Stable isotope labeling by metabolic incorporation of amino acids (SILAC) is mainly used for cell cultures, in the medium of which different stable isotopes are provided that will be incorporated into the proteins of the cells. At the point of analysis, cell extracts are mixed and then jointly investigated with mass spectrometry. Mass differences between peptide pairs due to the isotopic labeling can be exploited for relative quantitation [46]. Currently, up to three conditions (light, medium, heavy) can be multiplexed. Further developments of SILAC even produced an in-vivo SILAC mouse model for the proteomic and phosphoproteomic analysis of skin cancerogenesis [47] and superSILAC for the analysis of tissues [48], in which a metabolically labeled, tissue-specific protein mix from several cell lines, representing the complexity of the investigated proteome, is mixed with the tissue lysate as internal standard for quantification.

Chemical modification of peptides with tandem mass tags (TMT) or isobaric tags for relative and absolute quantitation (iTRAQ) are two different methods based on tags with reactive groups that bind to peptidyl amines in the peptides after protein digestion. Again, different samples are mixed before mass spectrometry analysis, whereas for TMT or iTRAQ up to eight samples can be multiplexed. In the first MS run, the peptides with different isobaric tags are indistinguishable, but upon fragmentation in the second MS run, each tag generates a unique reporter ion fragmentation spectrum, which can be used for relative quantitation of the tagged peptides $[49,50]$.

Label-free quantitation (LFQ), on the other hand, relies mainly on post-acquisition data analysis, so that no modification of the essential experimental workflow needs to be implemented. Comparison of an-in theory - unlimited number of different samples is therefore possible, which is associated with the downside of prolonged analysis time as multiplexing samples is not possible. While label-free approaches usually provide a deeper coverage of the proteome than label-based methods, the reproducibility and precision of quantification are inferior, so that more technical replicates 
2.4 Phosphosite Assignment are needed for confident quantification in LFQ [51]. Typically, label-free quantitation is achieved by integration of peak area measurements, i.e. the area under the curve, for individual peptides [52] or spectral counting, which reflects that the probability to sample more abundant peptides is higher [53].

For the case of phosphoproteomics, in contrast to regular proteomics, an additional challenge for quantitation arises from the fact that information from different peptides of the same protein cannot be integrated. While in regular proteomics the abundances of every peptide in the protein can be combined, the quantitation of a single phosphosite depends on direct measurements of peptides with the specific modification. Therefore, the sample sizes in phosphoproteomics quantitation are much smaller and can even consist of the measurement of only a single peptide [24].

Furthermore, different phosphosites within the same protein will in many cases not show similar pattern of phosphorylation dynamics. This may give rise to problems for subsequent analysis, if this analysis is conducted on protein rather than on phosphosite level.

Phosphopeptides in large-scale phosphoproteomics experiments are identified from LC-MS/MS runs by interpreting MS/MS spectra using a suitable search engine. Several of such search engines now exist; popular ones include Mascot, Sequest, Protein Prospector, and Andromeda [54-57]. The process of determining peptide sequences from MS / MS data involves matching the mass to charge ratios of fragment ions in MS/MS spectra to the theoretical fragmentation of all protein-derived peptides in protein databases. Depending on the organism being investigated, protein databases from UniProt or NCBI are used. Each search engine has its own scoring system to reflect the confidence of peptide identification, which is a function of MS and MS/MS spectral quality. The false discovery rate (FDR) may be determined by performing parallel searches against scrambled or reversed protein databases containing the same number of sequences as the authentic protein database. The FDR is then calculated as the ratio of positive peptide identifications in the decoy database divided by those derived from the forward search. An FDR of $1 \%$ at the peptide level is normally considered adequate.

Deriving peptide sequences with these methods is a relatively straightforward process. However, site localization can be a problem when peptide sequences contain more than one amino acid residue that can be phosphorylated. To address this problem, several methods to determine precise localization of phosphorylation within a phosphopeptide have been published. Ascore uses a probabilistic approach to assess correct site assignment [58] and the algorithm has been applied alongside the Sequest search engine. 


\subsection{Pitfalls in the Analysis of MS-Based Phospho- proteomics Data}

The Mascot delta score, introduced by the Kuster group, simply determines the differences in Mascot scores between the different possibilities for phosphosite localization within a phosphopeptide [59]. The larger the delta score, the greater the probability of correct site assignment. Other similar methods have been published [60] and some of them are now incorporated into search engines [61]. The output of the phosphopeptide identification step generally contains scores for both the probability of correct peptide sequence identification and phosphosite localization.

Although the available experimental methods for MS-based phosphoproteomics data acquisition have evolved considerably over the last years, leading to a steadily increasing number of detected phosphosites, several limitations remain for the investigation of signaling processes using phosphoproteomics data.

While it has been estimated that there are around 500,000 phosphorylation sites in the human proteome [62], the number of phosphosites that can be identified in a single MS experiment usually ranks around 10,000 to up to 40,000 [63]. Therefore, the sampled phosphoproteomic picture is incomplete. It has to be taken into account though, that, not all possible phosphorylation sites are expected to be modified at the same time point. This is caused by context-dependent regulation of phosphosites. For example, some phosphosites are controlled differentially at different cell cycle stages, while others only change under specific external stimulation such as growth factors or other effector molecules $[64,65]$. The hope is therefore that a significantly larger portion of phosphosites could be mapped with improving technology and by increasing the diversity of biologically relevant conditions analyzed. So far though, in different MS runs or replicates, usually a distinct set of phosphosites is detected, as the selection of precursor ions is stochastic. This leads to incomplete datasets with a high number of missing data points, challenging computational investigation of the data such as clustering or correlation analysis. However, as discussed above, approaches in which phosphopeptide intensities are compared across MS run post-acquisition minimize this problem [38].

The functional impact of a phosphorylation event is known only in the minority of cases [15]. Indeed, it has been hypothesized that a substantial fraction of phosphorylation sites are non-functional [66], since phosphorylation sites tend to be poorly conserved throughout species [67]. Although approaches to studying the function of individual phosphorylation events have been proposed [68], it may be that a large part of the detected phosphosites serves no function at all. Thus, non-functional sites add a substantial amount of noise to phosphoproteomics data and complicate the computational analysis.

The inference of kinase activity from phosphoproteomics data that will be described in the next section aims to overcome these limitations, by the integration of the information from many 
phosphosites, along prior knowledge on kinases-substrate relationships, into a single measure for the kinase activity. It is important though to keep in mind that any bias in the experimental workflow will affect these scores. In particular, since highly abundant precursor ions are more likely to be selected for fragmentation and therefore detection, targets of upregulated kinases are more probably detected. Therefore, highly active kinases will be preferentially detected, although downregulated kinases may be identified when comparing different conditions.

\section{Computational Methods for Inference of Kinase Activity}

Traditionally, biochemical methods have been common to study kinase activities in vitro and are still broadly used $[69,70]$. However, on the one hand those methods are generally limited in throughput and time-consuming. On the other hand in vitro methods might not accurately reflect the in vivo activities of kinases in a specific cellular context. MS-based methods have also been applied for assaying kinase activity $[9,10]$. Here, the abundances of known target phosphosites are monitored by MS after an in vitro enzymatic reaction.

Since every phosphorylation event results-by definitionfrom the activity of a kinase, phosphoproteomics data should be suitable to infer the activity of many kinases from a comparably low experimental effort. This task requires computational analysis of the detected phosphorylation sites (phosphosites), since thousands of phosphosites can routinely be measured in a single experiment. Several methods have been proposed in recent years, all of which utilize prior knowledge about kinase-substrate interactions, either from curated databases or from information about kinase recognition motifs.

3.1 Resources for Kinase-Substrate Relationships
As the large-scale detection of phosphorylation events using mass spectrometry became routine, many freely available databases that collect experimentally verified phosphosites have been set up, including PhosphoSitePlus [20], Phospho.ELM [21], Signor [71], or PHOSIDA [72], to name just a few. The databases differ in size and aim; PHOSIDA for example provides a tool for the prediction of putative phosphorylation sites and recently also added acetylation and other posttranslational modification sites to its scope. Phospho.ELM computes a score for the conservation of a phosphosite. Signor is focused on interactions between proteins participating in signal transduction. PhosphoNetworks [73] is dedicated to kinase-substrate interactions, but the information is on the level of proteins, not phosphosites. The arguably most prominent database for expert-edited and curated interactions between kinases and individual phosphosites (that have not been derived 
from in vitro studies) is PhosphoSitePlus, currently encompassing 16,486 individual kinase-substrate relationships [04-2015]. For Saccharomyces cerevisiae, the database PhosphoGRID provides analogous information [74]. Specific information about targets of phosphatases can be found in DEPOD [75]. Also in the Phospho. ELM database, phosphosites have been associated with regulating kinases, although this information is available for only about $10 \%$ of the 37,145 human phosphosites in the database [04-2015].

As it has been estimated that there are between 100,000 [76] and 500,000 [62] possible phosphosites in the human proteome, the evident low coverage of the curated databases motivated the development of computational tools to predict in vivo kinasesubstrate relationships. These methods identify putative new kinase-substrate relationships based on experimentally derived kinase recognition motifs, which was pioneered by Scansite [77] that uses position-specific scoring matrices (PSSMs) obtained by positional scanning of peptide libraries [78] or phage display methods [79]. Another approach, Netphorest [80] tries to classify phosphorylation sites according to the relevant kinase family instead of predicting individual kinase-substrate links. However, the in vitro specificity of kinases differs significantly from the kinase activity inside of the cell, biasing the experimentally identified kinase recognition motifs [81]. The integration of contextual information, for example co-expression, protein-protein interactions, or subcellular colocalization, markedly improves the accuracy of the predictions [69]. The software packages NetworKIN [82] (recently extended in the context of the resource KinomeXplorer [22], correcting for biases caused by over-studied proteins) and iGPS [23] are examples for methods that combine information about kinase recognition motifs, in vivo phosphorylation sites, and contextual information, e.g., from the STRING database [83]. Recently, Wagih et al. presented a method to predict kinase specificity for kinases without any known phosphorylation sites [84]. Based on the assumption that functional interaction partners of kinases (derived from the STRING database) are more likely to be phosphorylated by the respective kinase, they should therefore contain an amino acid motif conferring kinase specificity. This can then be uncovered by motif enrichment.

The described methods provide predictions that are very valuable but not free from error, for example due to the described differences in in vitro and in vivo kinase specificity or the influence of subcellular localization. Thus, the predicted kinase-substrate interactions should be considered hypotheses to be tested experimentally [85].

We hereafter present four computational methods to infer kinase activities from phosphoproteomics data, which use either curated or computationally predicted kinase-substrate interactions. 
3.2 GSEA

$3.3 \quad K A A$

Methodologically, inference of kinase activity from phosphoproteomics data is related to the inference of transcription factor activity based on gene expression data. A plethora of different methods has been developed for the prediction of transcription factor activity, e.g., the classical gene set enrichment analysis [86] or elaborated machine learning methods [87].

For example, Drake et al. [88] analyzed the kinase signaling network in castration-resistant prostate cancer with GSEA. They predicted the kinases responsible for each phosphosite with kinasesubstrate interactions from PhosphoSitePlus, kinase recognition motifs from PHOSIDA, and predictions from NetworKIN. Subsequently, they computed the enrichment of each kinase' targets with the gene set enrichment algorithm after Subramanian et al. [86], which corresponds to a Kolmogorov-Smirnov-like statistic. The significance of the enrichment score is determined based on permutation tests, whereas the $p$-value depends on the number of permutations.

Alternatively, the gene set enrichment web-tool Enrichr $[89,90]$ can also be used for enrichment of kinases [91]. The authors compiled kinases-substrate interactions from different databases and extracted additional interactions manually from the literature in order to generate kinase-targets sets. Furthermore, they added protein-protein interactions involving kinases from the Human Protein Reference Database (HPRD) [92], based on the assumption that those are highly enriched in kinase-substrate interactions. Using this prior knowledge, the enrichment of the targets of a kinase is then computed with Fisher's exact test as described in [89].

Another approach to link phosphoproteomics data with the activity of kinases was presented in a publication from Qi et al. [16], which they termed kinase activity analysis (KAA).

In this study, the authors collected phosphoproteomics data from adult mouse testis in order to investigate the process of mammalian spermatogenesis. With the software package iGPS [23] they predicted putative kinase-substrate relationships for the detected phosphosites. The authors hypothesized that the number of links for a given kinase in the predicted kinase-substrate network can serve as proxy for the activity of this kinase in the specific cell type. This activity was then compared to the kinase activity background which was calculated by computing the number of links in the background kinase-substrate network based on the mouse phosphorylation atlas by Huttlin et al. [93]. Qi and colleagues predicted high activity of PLK kinases in adult mouse testis and could validate this prediction experimentally.

However, there are several limitations of KAA. For once, it is mainly based on computational predictions of kinase substrate relationships, which are known to be susceptible to errors 
$[69,85]$. Additionally, in their method the activity of a kinase is only dependent on the number of detected, putative targets. The abundance of the individual phosphosites or the fold change between conditions is not taken into account.

De Graaf et al. [94] chose a comparable approach in a study of the phosphoproteome of Jurkat T cells after stimulation with prostaglandin $\mathrm{E}_{2}$. However, they did not explicitly calculate kinase activities. Instead, they grouped phosphosites into different clusters with distinct temporal profiles and used the NetworKIN algorithm [82] to calculate the enrichment of putative targets of a given kinase in a specific cluster. As a result, they associated kinases with temporal activity profiles based on the enrichment in one of the detected clusters.

\subsection{CLUE}

A method designed specifically for time-course phosphoproteomics data is the knowledge-based CLUster Evaluation approach, in short CLUE [18]. This method is based on the assumption that phosphosites targeted by the same kinase will show similar temporal profiles, which is utilized to guide a clustering algorithm and infer kinases associated with these clusters. As in the study by de Graaf et al. [94], kinases are not associated with distinct values for activities but rather with temporal activity profiles. The notable distinction of CLUE is that the clustering is found based on the prior knowledge about kinase-substrate relationships, as outlined below.

Methodologically, CLUE uses the $k$-means clustering algorithm to group the phosphoproteomics data into clusters in which the phosphosites show similar temporal kinetics. The performance of $k$-means clustering is particularly sensitive to the parameter $k$, i.e., the number of clusters. CLUE therefore tests a range of different values for $k$ and evaluates them based on the enrichment of kinase-substrate relationships in the identified clusters. The method utilizes the data from the PhosphoSitePlus database in order to derive prior knowledge about kinase-substrate relationships. With Fisher's exact test the enrichment of the targets of a given kinase in a specific cluster is tested for significance. The implemented scoring system penalizes distribution of the targets of a single kinase throughout several clusters, as well as the combination of unrelated phosphosites in the same cluster.

CLUE is freely available as $\mathrm{R}$ package in the Comprehensive $\mathrm{R}$ Archive Network CRAN under https://cran.r-project.org/web/ packages/ClueR/index.html.

A limitation of CLUE is represented by the fact that possible 'noise' in the prior knowledge, i.e., incorrect annotations, potentially derived from cell type-specific kinase-substrate relationships, can affect the performance of the clustering, although simulations showed reasonable robustness. CLUE is tailored toward timecourse phosphoproteomics data and associates kinases with 
3.5 KSEA

temporal activity profiles. Since the method does not provide singular activity scores for each kinase, it may be only partly applicable to experiments in which the individual responses of kinases to different treatments or conditions are of interest.

Casado et al. [17] presented a method for kinase activity estimation based on kinase-substrate sets. Using kinase-substrate relationships derived from the databases PhosphoSitePlus and Phospho.ELM, all phosphosites that are targeted by a given kinase can be grouped together into a substrate set (see Fig. I for an outline of the workflow). In theory, these phosphosites should show similar values, since they are targeted by the same kinase. However, due to the transient and therefore inherently noisy nature of phosphorylation, Casado and colleagues proposed integrating the information from all phosphosites in the substrate set in order to enhance the signalto-noise ratio by signal averaging [95].

For KSEA, $\log 2$-transformed fold change data is needed, i.e., the change of the abundance of a phosphosite between the initial and treated states, initial and later time points, or between two different cell types. Therefore, KSEA activity scores describe the activity of a kinase in one condition relative to another.

The authors suggested three possible metrics (mean score, alternative mean score, and delta score) that can be extracted out of the substrate set and serve as proxy for kinase activity: (1) The

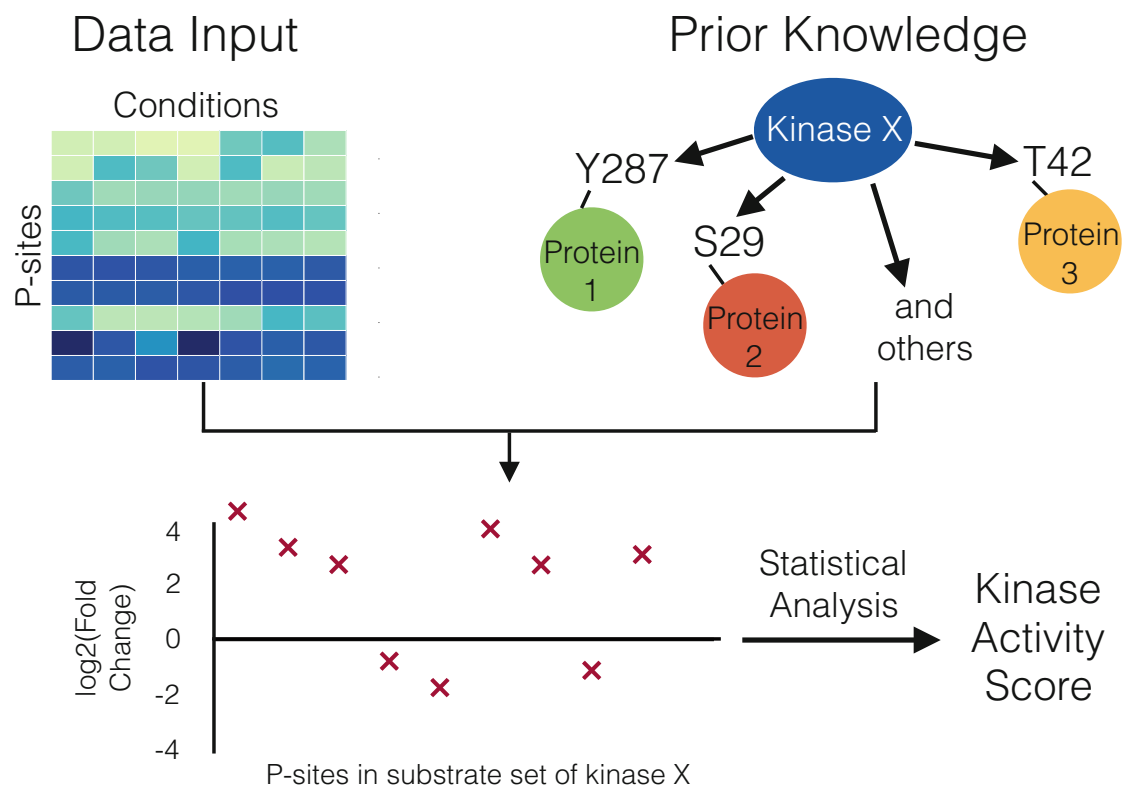

Fig. 1 Work-flow of methods to obtain Kinase activity scores such as KSEA. As prior knowledge, the targets of a given kinase are extracted out of curated databases like PhosphoSitePlus. Together with the data of the detected phosphosites, substrate sets are constructed for each kinase, from which an activity score can be calculated 
main activity score, also used in following publications [96], is defined as the mean of the $\log 2$ fold changes of the phosphosites in the substrate set; (2) alternatively, only phosphosites with significant fold changes can be considered for the calculation of the mean; and ( 3 ) for the last approach, termed "delta count," the occurrence of significantly upregulated phosphosites in the substrate set is counted, from which the number of significantly downregulated sites is subtracted. For each method, the significance of the kinase activity score is tested with an appropriate statistical test. In the publication of Casado et al., all three measures were in good agreement, even if spanning different numerical ranges (see Fig. 2). The implementation of these three methods is discussed in detail in the following section.

Like the other methods described in this section, KSEA strongly depends on the prior knowledge kinase-substrate relationships available in the freely accessible databases. These are far from complete and therefore limit the analytical depth of the kinase activity analysis. Additionally, databases are generally biased toward well-studied kinases or pathways [22], so that the sizes of the different substrate sets differ considerably. Casado et al. tried to address these limitations by integrating information about kinase recognition motifs and obtained comparable results.

A detailed protocol on how to use KSEA is provided in Subheading 4 .

Recently, Mischnik and colleagues introduced a machine-learning method to estimate kinase activities and to predict putative kinasesubstrate relationships from phosphoproteomics data [19].

In their model for kinase activity, the effect $e$ of a given kinase $j$ on a single phosphosite $i$ is modeled with

$$
e_{j i}=k_{j} \times p_{j i}
$$

as a product of the kinase activity $k$ and the affinity $p$ of kinase $j$ for phosphosite $i$. The abundance $P$ of the phosphosite $i$ is expressed as mean of all effects acting on it, since several kinases can regulate the same phosphosite:

$$
P_{i}=\sum_{j=1}^{m} e_{j i} / \sum_{j=1}^{m} p_{j i}
$$

The information about the kinase-substrate relationships is also derived from the PhosphoSitePlus database. Using a nonlinear optimization routine, IKAP estimates the described parameters while minimizing a least square cost function between predicted and measured phosphosite abundance throughout time points or conditions. For this optimization, the affinity parameters are estimated globally, while the kinase activities are fitted separately for each time point. 
A

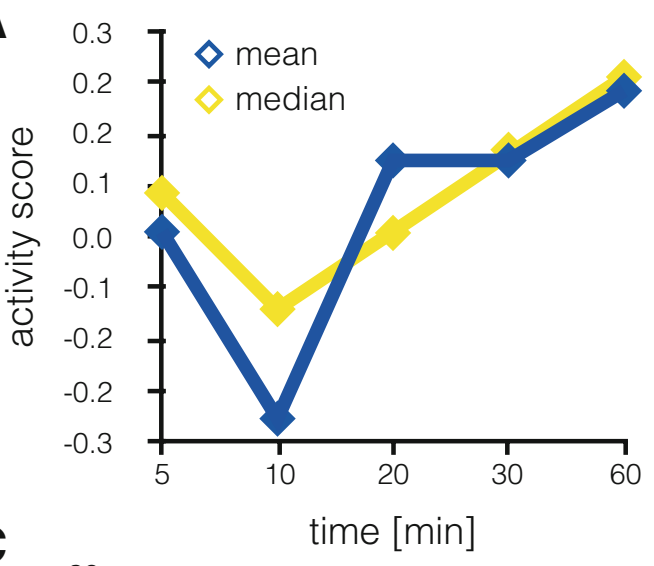

C

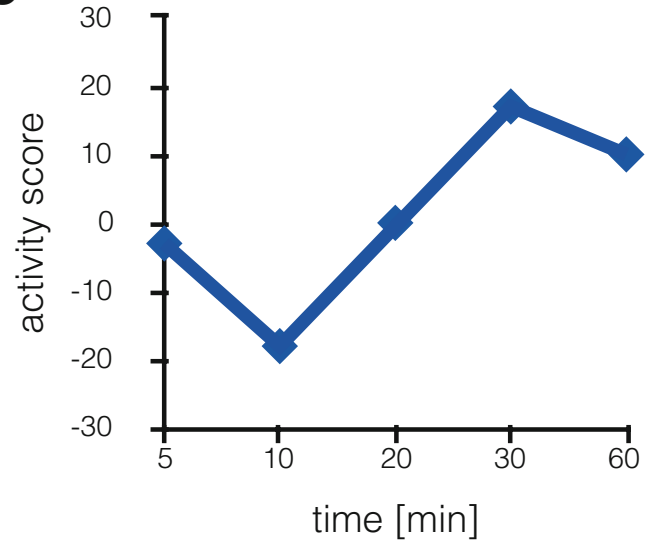

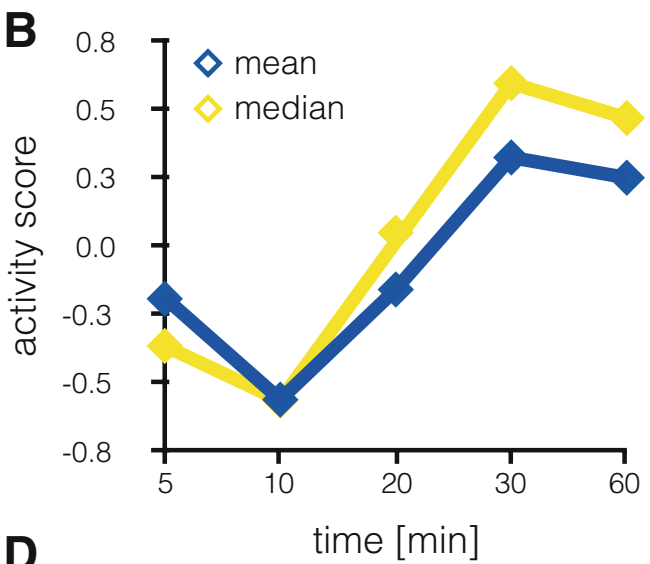

D

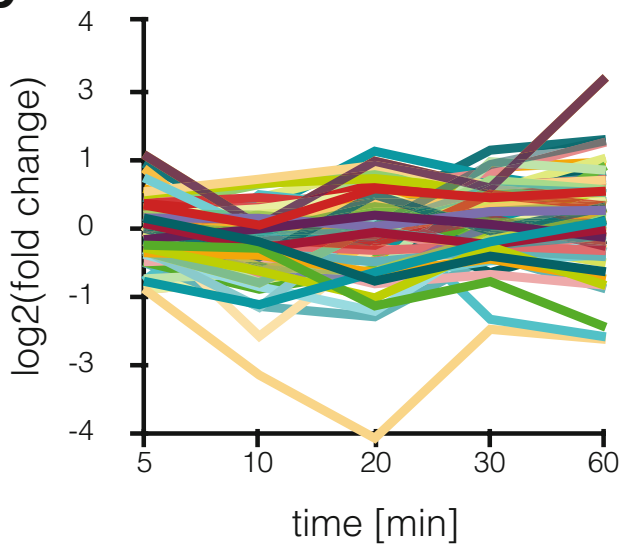

Fig. 2 KSEA activity scores for Casein kinase II subunit alpha. (a) Activity scores for Casein kinase II subunit alpha over all time points of the de Graaf dataset [94], calculated as the mean of all phosphosites in the substrate set. In yellow, the median has been used. (b) Activity scores for Casein kinase II subunit alpha over all time points of the de Graaf dataset, calculated as the mean of all significantly regulated phosphosites in the substrate set. The median is again shown in yellow. (c) Delta score for Casein kinase II subunit alpha over all time points of the de Graaf dataset, calculated as number of significantly upregulated phosphosites minus the number of significantly downregulated phosphosites in the substrate set. (d) The log2 fold changes for all time points for all phosphosites in the substrate set of the Casein kinase II subunit alpha

In a second step, putative new kinase-substrate relationships are predicted based on the correlation of a phosphosite with the estimated activity of a kinase throughout time points or conditions. These predictions are then tested by database searches and by comparison to kinase recognition motifs from NetworKIN.

In contrast to KSEA, which computes the kinase activity based on the fold changes of the phosphosites in the respective substrate set, IKAP is built on a heuristic machine learning algorithm and tries to fit globally the described model of kinase activity and affinity to the phosphoproteomics data. Therefore, the output of IKAP is not only a score for the activity of a kinase, but also a value representing the strength of a specific kinase-substrate interaction 
in the investigated cell type. On the other hand, the amount of parameters that have to be estimated is rather large, so that a fair number of experimental conditions or time points are needed for unique solutions. Mischnik et al. included a function to perform an identifiability analysis of the obtained kinase activities and could show in the case of the two investigated example datasets that the found solutions are indeed unique on the basis of the phosphoproteomics measurements.

The MATLAB code for IKAP can be found online under www. github.com/marcel-mischnik/IKAP/, accompanied by an extensive step-by-step documentation, which we recommend as additional reading to the interested reader.

\section{Protocol for KSEA}

\subsection{Quick Start}

In this section, we present a stepwise, guided protocol for the KSEA approach to infer kinase activities from phosphoproteomics data. This protocol (part of the Kinase Activity Toolbox under https://github.com/saezlab/kinact) is accompanied by a freely available script, written in the Python programming language (Python version 2.7.x) that should enable the use of KSEA for any phosphoproteomics dataset. We plan to expand Kinact to other methods in the future. We are going to explain the performed computations in detail in the following protocol to facilitate understanding and to enable a potential re-implementation into other programming languages.

As an example application, we will use KSEA on the phosphoproteomics dataset from de Graaf et al. [94], which was derived from Jurkat $T$ cells stimulated with prostaglandin $\mathrm{E}_{2}$ and is available as supplemental information to the article online at http://www. mcponline.org/content/13/9/2426/suppl/DCl

As a quick start for practiced Python users, we can use the utility functions from kinact to load the example dataset. The data should be organized as Pandas DataFrame containing the $\log 2$ transformed fold changes, while the columns represent different conditions or time points and the row individual phosphosites. The $p$-value of the fold change is optional, but should be organized in the same way as the data.

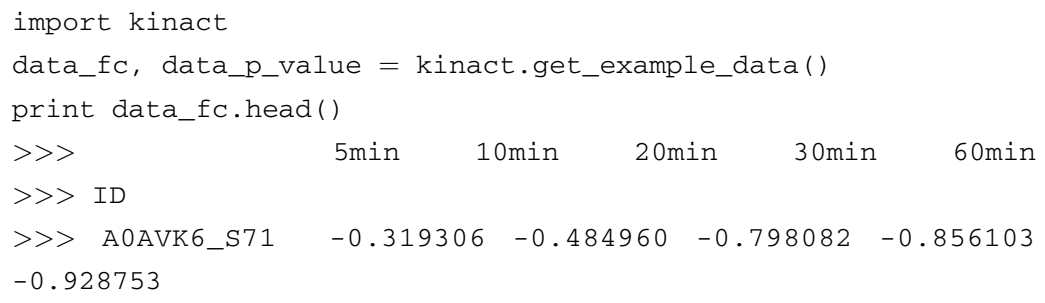




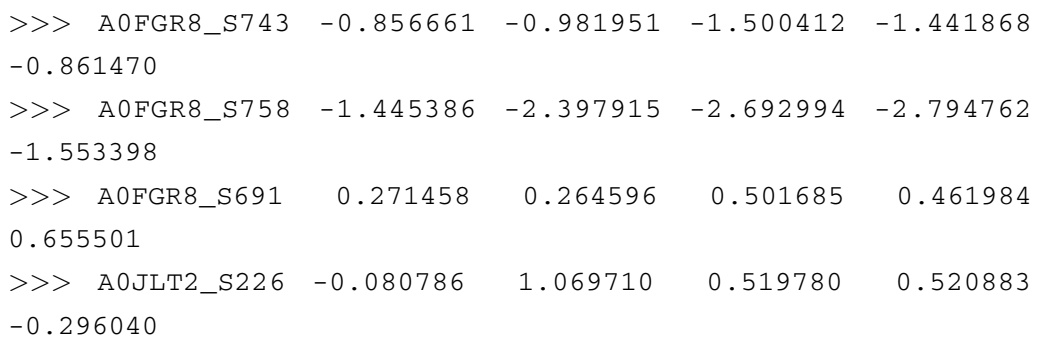

The kinase-substrate relationships have to be loaded as well with the function get_kinase_targets(). In this function call, we can specify with the 'sources'-parameter, from which databases we want to integrate the information about kinase-substrate relationships, e.g., PhosphoSitePlus, Phospho.ELM, or Signor. The function uses an interface to the pypath python package, which integrates several resources for curated signaling pathways [97] ( see also Note $\mathbf{1})$.

kin_sub_interactions $=$ kinact.get_kinase_targets $($ sources $=$ $\left[{ }^{\prime} a l 1 '\right]$ )

An important requirement for the following analysis is that the structure of the indices of the rows of the data and the prior knowledge need to be the same (see below for more detail). As an example, KSEA can be performed for the condition of 5 min after stimulation in the de Graaf dataset using:

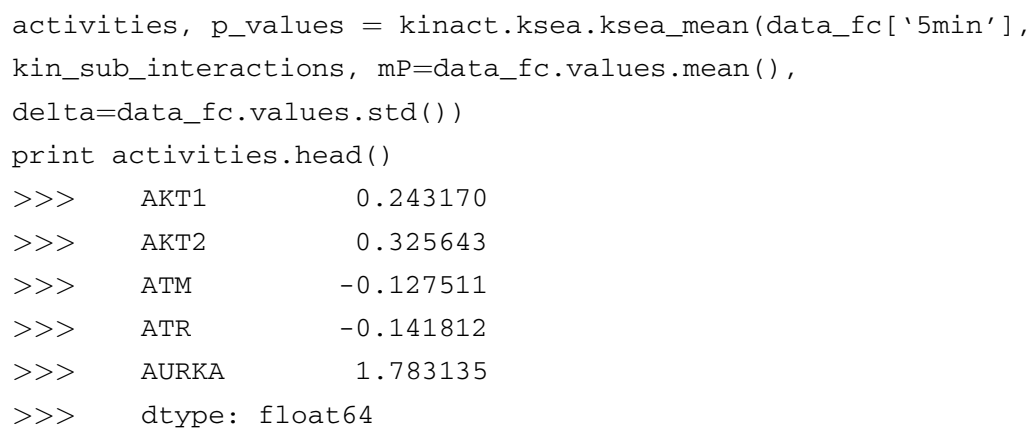

Besides the data (data_fc[' $5 \mathrm{~min}$ ']) and kinase-substrate interactions (kin_sub_interactions), the variables ' $\mathrm{mP}$ ' and 'delta' are needed to determine the $z$-score of the enrichment. The $z$-score builds the basis for the $p$-value calculation. The $p$-values for all kinases are corrected for multiple testing with the BenjaminiHochberg procedure [98].

In Fig. 2, the different activity scores for the Casein kinase II alpha, which de Graaf et al. had associated with increased activity after prolonged stimulation with prostaglandin $\mathrm{E}_{2}$, are shown together with the $\log 2$ fold change values of all phosphosites that 
are known to be targeted by this kinase. For methods, which use the mean, the median as more robust measure can be calculated alternatively. The qualitative changes of the kinase activities (Fig. $2 \mathrm{a}-\mathrm{c}$ ) are quite similar regardless of the method, and would not be apparent from looking at any specific substrate phosphosite alone (Fig. 2d).

4.2 Loading the Data

In the following, we walk the reader step by step through the procedure for KSEA. First, we need to organize the data so that the KSEA functions can interpret it.

In Python, the library Pandas [99] provides useful data structures and powerful tools for data analysis. Since the provided script depends on many utilities from this library, we would strongly advice the reader to have a look at the Pandas documentation, although it will not be crucial in order to understand the presented protocol. The library, together with the NumPy [100] package, can be loaded with:

import pandas as pd

import numpy as np

The data accompanying the article is provided as Excel spreadsheet and can be imported to python using the pandas 'read_excel' function or first be saved as csv-file, using the 'Save As' function in Excel in order to use it as described below. For convenience, in the referenced Github repository, the data is already stored as csv-file, so that this step is not necessary. The data can be loaded with the function 'read_csv', which will return a Pandas DataFrame containing the data organized in rows and columns.

data_raw $=$ pd.read_cSV('FILEPATH', $\left.\operatorname{sep}={ }^{\prime},{ }^{\prime}\right)$

In the DataFrame object 'data_raw', the columns represent the different experimental conditions or additional information and the row's unique phosphosites. A good way to gain an overview about the data stored in a DataFrame and to keep track of changes are the following functions:

print data_raw.head() to show the first five rows of the DataFrame or print data_raw.shape in order to show the dimensions of the DataFrame.

Phosphosites that can be matched to different proteins or several positions within the same protein are excluded from the analysis. In this example, ambiguous matching is indicated by the presence of a semicolon that separates multiple possible identifiers, and can be removed like this:

data_reduced $=$ data_raw[ data_raw['Proteins'].str.contains $\left.\left({ }^{\prime}{ }^{\prime}\right)\right]$ 
For more convenient data handling, we will index each phosphosite with an unambiguous identifier comprising the UniProt accession number, the type of the modified residue, and the position within the protein. For the example of a phosphorylation of the serine 59 in the Tyrosine-protein kinase Lck, the identifier would be P06239_S59. The identifier can be constructed by concatenating the information that should be provided in the dataset. In the example of de Graaf et al., the UniProt accession number can be found in the column 'Proteins', the modified residue in 'Amino acid', and the position in 'Positions within proteins'.

The index is used to access the rows in a DataFrame and will later be needed to construct the kinase-substrate sets. After the creation of the identifier, the DataFrame is indexed by calling the function 'set_index'.

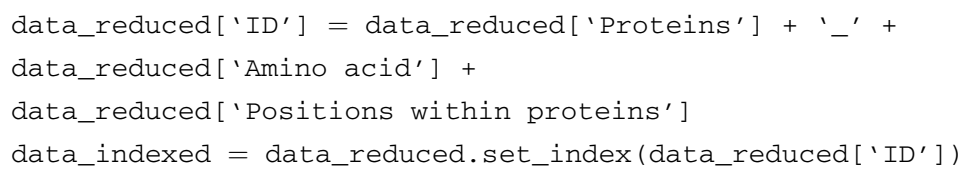

Mass spectrometry data is usually accompanied by several columns containing additional information about the phosphosite (e.g., the sequence window) or statistics of the database search (for example the posterior error probability), which are not necessarily needed for KSEA. We therefore extract only the columns of interest containing the processed data. In the example dataset, the names of the crucial columns start with 'Average', enabling selection by a simple 'if' statement. Generally, more complex selection of column names can be achieved by regular expressions with the python module 're'.

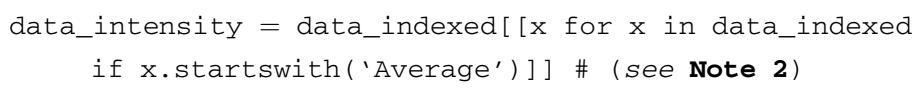

Now, we can compute the fold change compared to the control, which is the condition of 0 min after stimulation. With $\log (a /$ $b)=\log (a)-\log (b)$, we obtain the fold changes by subtracting the column with the control values from the rest using the 'sub' function of Pandas ( see Note 3).

data_fc $=$ data_intensity.sub(data_intensity['Average Log2 Intensity $\left.0 \mathrm{~min}^{\prime}\right]$, axis=0)

Further data cleaning (re-naming columns and removal of the columns for the control time point) results in the final dataset:

data_fc.columns $=[x . s p l i t()[-1]$ for $x$ in data_fc $]$ \# Rename columns 


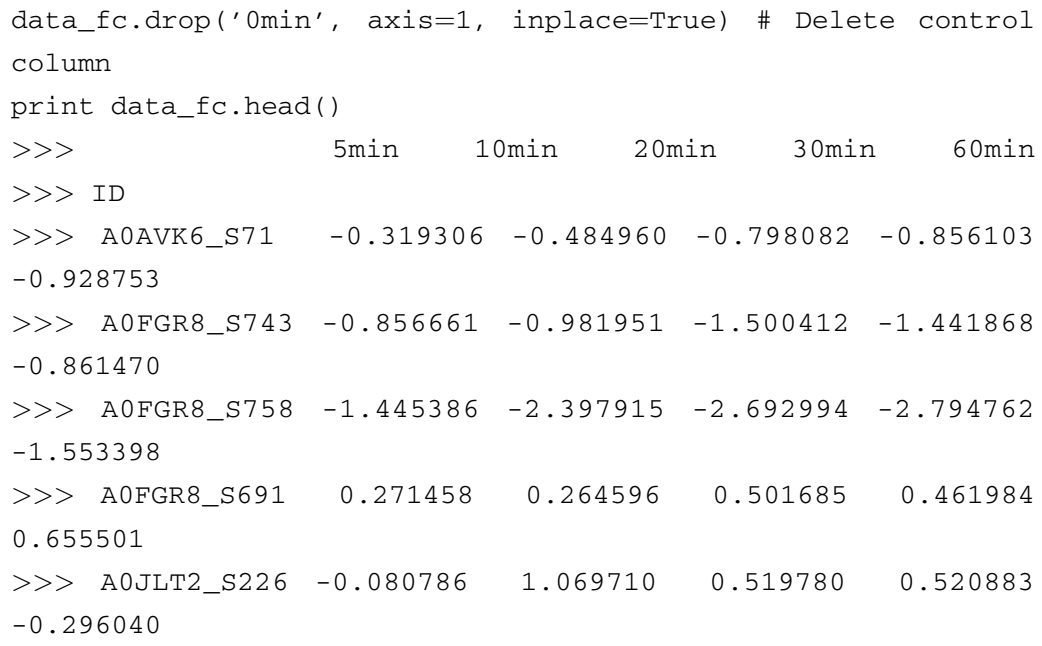

If the experiments have been performed with several replicates, statistical analysis enables estimation of the significance of the fold change compared to a control expressed by a $p$-value. The $p$-value will be needed to perform KSEA using the 'Delta count' approach but may be dispensable for the mean methods. The example dataset contains a $p$-value (transformed as negative logarithm with base 10) in selected columns and can be extracted using:

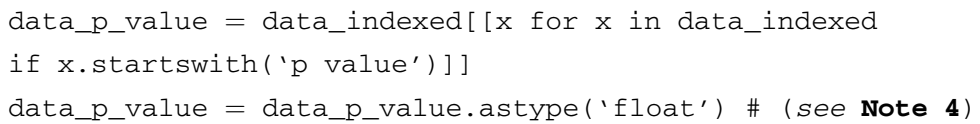

\subsection{Loading the Kinase-Substrate Relationships}

Now, we load the prior knowledge about kinase-substrate relationships. In this example, we use the information provided in the PhosphoSitePlus database ( see Note 5), which can be downloaded from the website www.phosphosite.org. The organization of the data from comparable databases, e.g., Phospho.ELM, does not differ drastically from the one from PhosphoSitePlus and therefore requires only minor modifications. Using 'read_csv' again, we load the downloaded file with:

ks_rel $=$ pd.read_csv('FILEPATH', $\left.\operatorname{sep}=^{\prime} \backslash t^{\prime}\right) \#($ see Note 6)

In this file, every row corresponds to an interaction between a kinase and a unique phosphosite. However, it must first be restricted to the organism of interest, e.g., 'human' or 'mouse', since the interactions of different organisms are reported together in PhosphoSitePlus.

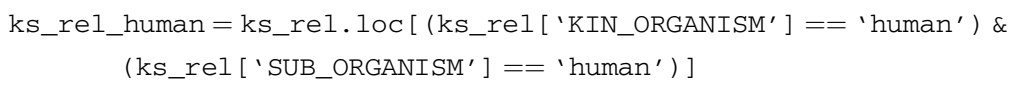


Next, we again construct unique identifiers for each phosphosite using the information provided in the dataset. The modified residue and its position are already combined in the provided data.

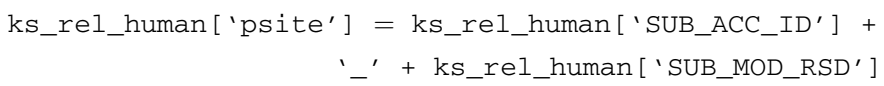

Now, we construct an adjacency matrix for the phosphosites and the kinases. In this matrix, an interaction between a kinase and a phosphosite is denoted with a $l$, all other fields are filled with a 0 . For this, the Pandas function 'pivot_table' can be used:

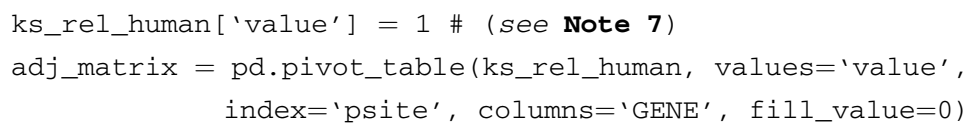

The result is an adjacency matrix of the form $m \times n$ with $m$ being the number of phosphosites and $n$ the number of kinases. If a kinase is known to phosphorylate a given phosphosite, the corresponding entry in this matrix will be a 1 , otherwise a 0 . A 0 does not mean that there cannot be an interaction between the kinase and the respective phosphosite, but rather that this specific interaction has not been reported in the literature. As sanity check, we can print the number of known kinase-substrate interactions for each kinase saved in the adjacency matrix:

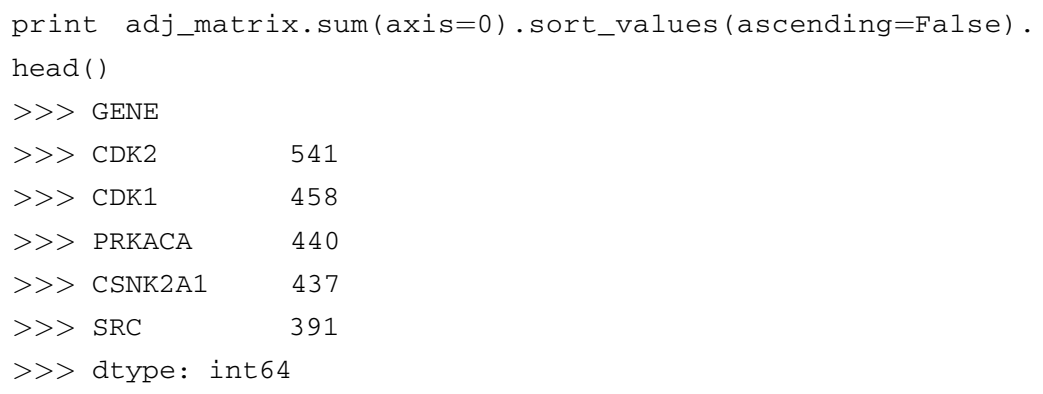

In the accompanying toolbox, we provide for each method of KSEA a custom python function that automates the analysis for all kinases in a given condition. Here, however, we demonstrate the principle of KSEA by computing the different activity scores for a single kinase and a single condition. As an example, the Cyclindependent kinase 1 (CDKl, see Note 8) and the condition of $60 \mathrm{~min}$ after prostaglandin stimulation shall be used.

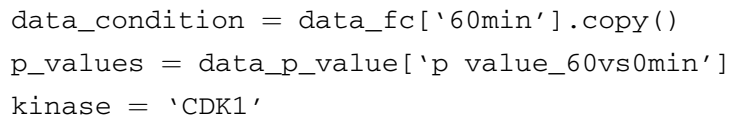


First, we determine the overlap between the known targets of the kinase and the detected phosphosites in this condition, because we need it for every method of KSEA. Now, we benefit from having the same format for the index of the dataset and the adjacency matrix. We can use the Python function 'intersection' to determine the overlap between two sets.

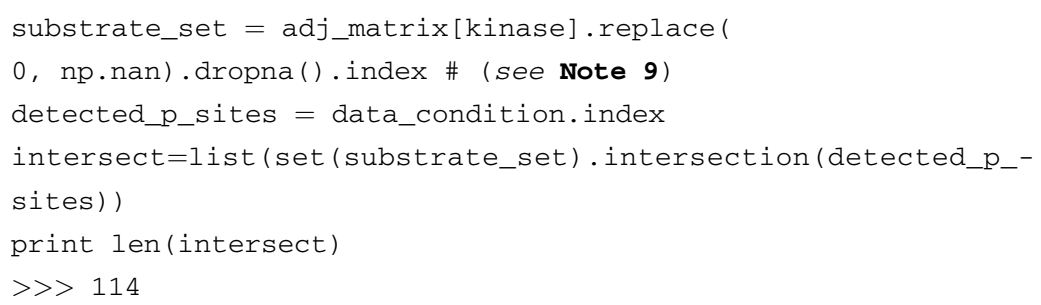

4.4.1 KSEA Using the "Mean" Method
4.4.2 KSEA Using the Alternative 'Mean' Method
For the "mean" method, the KSEA score is equal to the mean of the fold changes in the substrate set $m S$.

The significance of the score is tested with a $z$-statistic using

$$
z=\frac{m S-m P \sqrt{m}}{\delta}
$$

with $m P$ as mean of the complete dataset, $m$ being the size of the substrate set, and $\delta$ the standard deviation of the complete dataset, adapted from the PAGE method for gene set enrichment [101]. The "mean" method has established itself as the preferred method in the Cutillas lab that developed the KSEA approach.

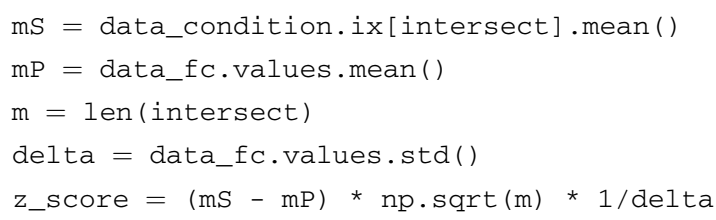

The $z$-score can be converted into a $p$-value with a function from the SciPy [102] library:

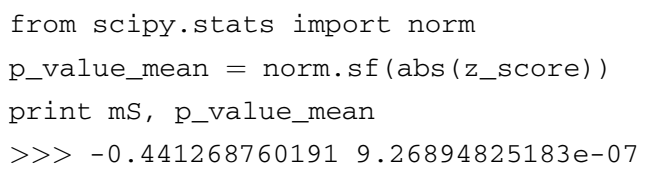

Alternatively, only the phosphosites in the substrate set that change significantly between conditions can be considered when computing the mean of the fold changes in the substrate set. Therefore, we need a cutoff, determining a significant increase or decrease, respectively, which can be a user-supplied parameter. Here, we use a 
4.4.3 KSEA Using the "Delta Count" Method

standard level to define a significant change with a cutoff of 0.05 . The significance of the KSEA score is tested as before with the zstatistic.

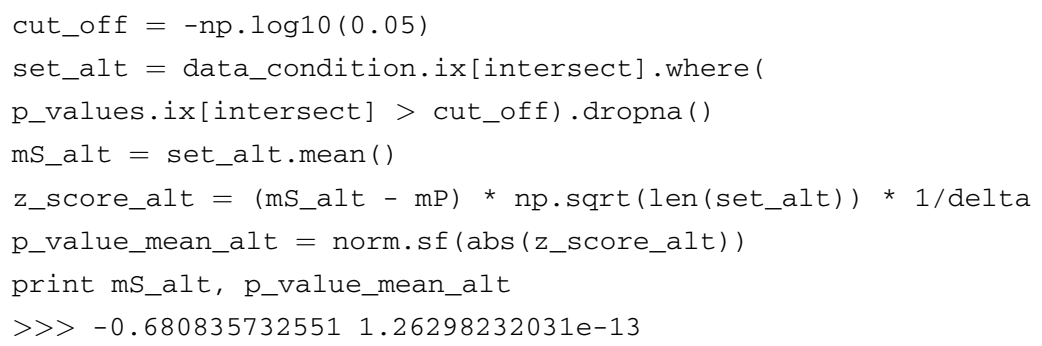

In the "Delta count" method, we count the number of phosphosites in the substrate set that are significantly increased in the condition versus the control and subtract the number of phosphosites that are significantly decreased.

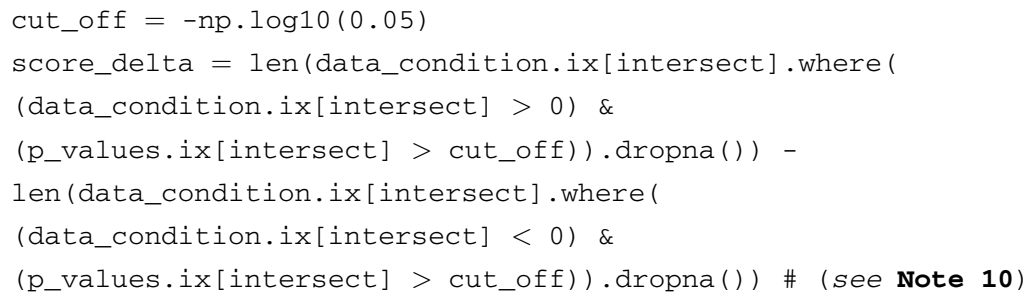

The $p$-value of the score is calculated with a hypergeometric test, since the number of significantly regulated phosphosites is a discrete variable. To initialize the hypergeometric distribution, we need as variables $M=$ the total number of detected phosphosites, $n=$ the size of the substrate set, and $N=$ the total number of phosphosites that are in an arbitrary substrate set and significantly regulated.

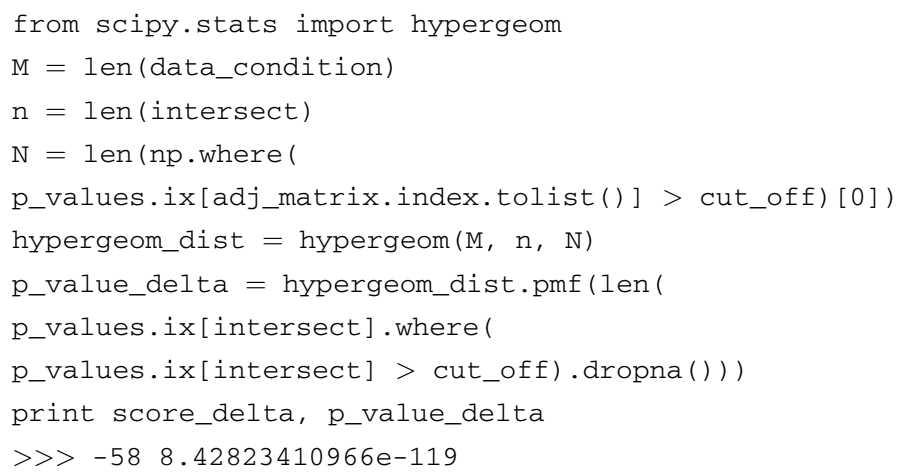


In summary, the methods described in this review use different approaches to calculate kinase activities or to relate kinases to activity profiles from phosphoproteomics datasets. All of them utilize prior knowledge about kinase-substrate relationships, either from curated databases or from computational prediction tools. Using these methods, the noisy and complex information from the vast amount of detected phosphorylation sites can be condensed into a much smaller set of kinase activities that is easier to interpret. Modeling of signaling pathways or prediction of drug responses can be performed in a straightforward way with these kinase activities as shown in the study by Casado et al. [17].

The power of the described methods strongly depends on the available prior knowledge about kinase-substrate relationships. As our knowledge increases due to experimental methods like in vitro kinase selectivity studies [103] or the CEASAR (Connecting Enzymes And Substrates at Amino acid Resolution) approach [104], the utility and applicability of methods for inference of kinase activities will grow as well. Additionally, the computational approaches for the prediction of possible kinase-substrate relationships are under on-going development [84, 105], increasing the reliability of the in silico predictions.

Phosphoproteomic data is not only valuable for the analysis of kinase activities: for example, PTMfunc is a computational resource that predicts the functional impact of posttranslational modifications based on structural and domain information [15], and PHONEMeS $[96,106]$ combines phosphoproteomics data with prior knowledge kinase-substrate relationships, in a similar fashion as kinase-activity methods. However, instead of scoring kinases, PHONEMeS derives logic models for signaling pathways at the phosphosite level.

For the analysis of deregulated signaling in cancer, mutations in key signaling molecules can be of crucial importance. Recently, Creixell and colleagues presented a systematic classification of genomic variants that can perturb signaling, either by rewiring of the signaling network or by the destruction of phosphorylation sites [107]. Another approach was introduced in the last update of the PhosphoSitePlus database, in which the authors reported with PTMVar [20] the addition of a dataset that can map missense mutation onto the posttranslational modifications. With these tools, the challenging task of creating an intersection between genomic variations and signaling processes may be addressed.

It remains to be seen how the different scoring metrics for kinase activity relate to each other, as they utilize different approaches to extract a kinase activity score out of the data. IKAP is based on a nonlinear optimization for the model of kinase- 
dependent phosphorylation, KSEA on statistical analysis of the values in the substrate set of a kinase, and CLUE on the $k$-means clustering algorithm together with Fisher's exact test for enrichment. In a recent publication by Hernandez-Armenta et al. [108], the authors compiled a benchmark dataset from the literature, consisting of phosphoproteomic experiments under perturbation. For each experiment, specific kinases are expected to be regulated, e.g., EGFR receptor tyrosine kinase after stimulation with EGF. Using this "gold standard," the authors assessed how well different methods for the inference of kinase activities could recapitulate the expected kinase regulation in the different conditions. All of the assessed methods performed comparably strongly, but the authors observed a strong dependency on the prior knowledge about kinase-substrate relationships. This is a first effort to assess the applicability, performance, and drawbacks of the different methods, thereby guiding the use of phosphoproteomics data to infer kinase activities, from which to derive insights into molecular cancer biology and many other processes controlled by signal transduction.

\section{Notes}

1. To the sources parameter in the function get_kinase_targets, either a list of kinase-substrate interaction sources that are available in pypath or 'all' in order to include all sources can be passed. If no source is specified, only the interactions from PhosphoSitePlus and Signor will be used. The available sources in pypath are "ARN" (Autophagy Regulatory Network) [109], "CAl" (Human Hippocampal CAl Region Neurons Signaling Network) [110], "dbPTM" [111], "DEPOD" [75], "HPRD" (Human Protein Reference Database) [92], "MIMP" (Mutation IMpact on Phosphorylation) [112], "Macrophage" (Macrophage pathways) [113], "NRF2ome" [114], "phosphoELM" [21], "PhosphoSite" [20], "SPIKE" (Signaling Pathway Integrated Knowledge Engine) [115], "SignaLink3" [116], "Signor" [71], and "TRIP" (Mammalian Transient Receptor Potential Channel-Interacting Protein Database) [117].

2. The provided code is equivalent to:

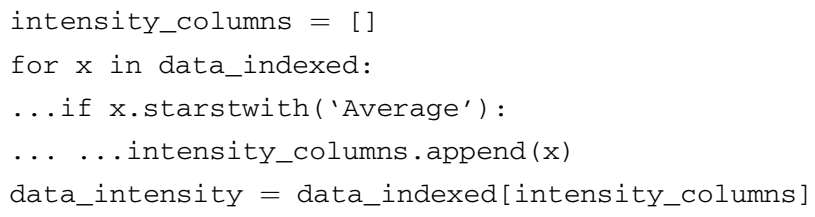


3. In our example, it is not necessary to transform the data to $\log 2$ intensities, since the data is already provided after $\log 2$ transformation. But for raw intensity values, the following function from the NumPy module can be used:

data_log2 = np. $\log 2$ (data_intensity)

4. Due to a compatibility problem with the output of Excel, Python recognizes the $p$-values as string variables, not as floating point numbers. Therefore, this line is needed to convert the type of the $p$-values.

5. The adjacency matrix can also be constructed based on kinase recognition motifs or kinase prediction scores and the amino acid sequence surrounding the phosphosite. To use NetworKIN scores for the creation of the adjacency matrix, kinact will provide dedicated functions. In the presented example, however, we focus on the curated kinase-substrate relationships from PhosphoSitePlus.

6. The file from PhosphoSitePlus is provided as text file in which a tab (' $\backslash \mathrm{t}$ ') delimits the individual fields, not a comma. The file contains a disclaimer at the top, which has to be removed first. Alternatively, the option 'skiprows' in the function 'read_csv' can be set in order to ignore the disclaimer.

7. This column is needed, so that in the matrix resulting from pd. pivot_table the value from this column will be entered.

8. If necessary, mapping between protein names, gene names, and UniProt-Accession numbers can easily be performed with the Python module 'bioservices', to the documentation of which we want the refer the reader [118].

9. In this statement, we first select the relevant columns of the kinase from the connectivity matrix (adj_matrix[kinase]). In this column, we replace all 0 values with $\mathrm{NAs}$ (replace $(0, \mathrm{np}$. nan)), which are then deleted with dropna(). Therefore, only those interactions remain, for which a $l$ had been entered in the matrix. Of these interactions, we extract the index, which is a list of the phosphosites known to be targeted by the kinase of interest.

10. The where method will return a copy of the DataFrame, in which for cases where the condition is not true, NA is returned. dropna will therefore delete all those occurrences, so that len will count how often the condition is true. 
Acknowledgments

Thanks to Emanuel Gonçalves, Aurélien Dugourd, and Claudia Hernández-Armenta for comments on the manuscript. For help with the code, thanks to Emanuel Gonçalves.

\section{References}

1. Jørgensen C, Linding R (2010) Simplistic pathways or complex networks? Curr Opin Genet Dev 20:15-22

2. Hanahan D, Weinberg RA (2011) Hallmarks of cancer: the next generation. Cell 144:646-674

3. Sawyers CL (1999) Chronic myeloid leukemia. N Engl J Med 340:1330-1340

4. Sawyers CL, Hochhaus A, Feldman E et al (2002) Imatinib induces hematologic and cytogenetic responses in patients with chronic myelogenous leukemia in myeloid blast crisis: results of a phase II study. Blood 99:3530-3539

5. Zhang J, Yang PL, Gray NS (2009) Targeting cancer with small molecule kinase inhibitors. Nat Rev Cancer 9:28-39

6. Gonzalez de Castro D, Clarke PA, Al-Lazikani B et al (2012) Personalized cancer medicine: molecular diagnostics, predictive biomarkers and drug resistance. Clin Pharmacol Ther 93:252-259

7. Cutillas PR (2015) Role of phosphoproteomics in the development of personalized cancer therapies. Proteomics Clin Appl 9:383-395

8. Bertacchini J, Guida M, Accordi B et al (2014) Feedbacks and adaptive capabilities of the PI3K/Akt/mTOR axis in acute myeloid leukemia revealed by pathway selective inhibition and phosphoproteome analysis. Leukemia 28:2197-2205

9. Cutillas PR, Khwaja A, Graupera $M$ et al (2006) Ultrasensitive and absolute quantification of the phosphoinositide 3-kinase/Akt signal transduction pathway by mass spectrometry. Proc Natl Acad Sci U S A 103:8959-8964

10. Yu Y, Anjum R, Kubota K et al (2009) A sitespecific, multiplexed kinase activity assay using stable-isotope dilution and high-resolution mass spectrometry. Proc Natl Acad Sci U S A 106:11606-11611

11. McAllister FE, Niepel M, Haas W et al (2013) Mass spectrometry based method to increase throughput for kinome analyses using ATP probes. Anal Chem 85:4666-4674
12. Doll S, Burlingame AL (2015) Mass spectrometry-based detection and assignment of protein posttranslational modifications. ACS Chem Biol 10:63-71

13. Choudhary C, Mann M (2010) Decoding signalling networks by mass spectrometrybased proteomics. Nat Rev Mol Cell Biol 11:427-439

14. Sabidó E, Selevsek N, Aebersold R (2012) Mass spectrometry-based proteomics for systems biology. Curr Opin Biotechnol 23:591-597

15. Beltrao P, Albanèse V, Kenner LR et al (2012) Systematic functional prioritization of protein posttranslational modifications. Cell 150:413-425

16. Qi L, Liu Z, Wang J et al (2014) Systematic analysis of the phosphoproteome and kinasesubstrate networks in the mouse testis. Mol Cell Proteomics 13:3626-3638

17. Casado P, Rodriguez-Prados J-C, Cosulich $S C$ et al (2013) Kinase-substrate enrichment analysis provides insights into the heterogeneity of signaling pathway activation in leukemia cells. Sci Signal 6:rs6

18. Yang P, Zheng X, Jayaswal V et al (2015) Knowledge-based analysis for detecting key signaling events from time-series Phosphoproteomics data. PLoS Comput Biol 11: el004403

19. Mischnik M, Sacco F, Cox J et al (2015) IKAP: a heuristic framework for inference of kinase activities from Phosphoproteomics data. Bioinformatics 32(3):424-431

20. Hornbeck PV, Zhang B, Murray B et al (2015) PhosphoSitePlus, 2014: mutations, PTMs and recalibrations. Nucleic Acids Res 43:D512-D520

21. Dinkel H, Chica C, Via A et al (2011) Phospho.ELM: a database of phosphorylation sites-update 2011. Nucleic Acids Res 39: D261-D267

22. Horn H, Schoof EM, Kim J et al (2014) KinomeXplorer: an integrated platform for kinome biology studies. Nat Methods 11:603-604 
23. Song C, Ye M, Liu Z et al (2012) Systematic analysis of protein phosphorylation networks from phosphoproteomic data. Mol Cell Proteomics 11:1070-1083

24. Riley NM, Coon JJ (2016) Phosphoproteomics in the age of rapid and deep proteome profiling. Anal Chem 88:74-94

25. Nilsson CL (2012) Advances in quantitative phosphoproteomics. Anal Chem 84:735-746

26. Hennrich ML, Gavin A-C (2015) Quantitative mass spectrometry of posttranslational modifications: keys to confidence. Sci Signal 8:re5

27. Giansanti P, Aye TT, van den Toorn $\mathrm{H}$ et al (2015) An augmented multiple-proteasebased human phosphopeptide atlas. Cell Rep 11:1834-1843

28. Ruprecht B, Roesli C, Lemeer S et al (2016) MALDI-TOF and nESI Orbitrap MS/MS identify orthogonal parts of the phosphoproteome. Proteomics 16(10):1447-1456

29. Zhou H, Ye M, Dong J et al (2013) Robust phosphoproteome enrichment using monodisperse microsphere-based immobilized titanium (IV) ion affinity chromatography. Nat Protoc 8:461-480

30. Rush J, Moritz A, Lee KA et al (2005) Immunoaffinity profiling of tyrosine phosphorylation in cancer cells. Nat Biotechnol 23:94-101

31. Ruprecht B, Koch H, Medard G et al (2015) Comprehensive and reproducible phosphopeptide enrichment using iron immobilized metal ion affinity chromatography (Fe-IMAC) columns. Mol Cell Proteomics 14:205-215

32. Domon B, Aebersold R (2006) Mass spectrometry and protein analysis. Science (New York, NY) 312:212-217

33. Nesvizhskii AI (2007) Protein identification by tandem mass spectrometry and sequence database searching. Methods Mol Biol (Clifton, NJ) 367:87-119

34. Liu H, Sadygov RG, Yates JR (2004) A model for random sampling and estimation of relative protein abundance in shotgun proteomics. Anal Chem 76:4193-4201

35. Cutillas PR, Vanhaesebroeck B (2007) Quantitative profile of five murine core proteomes using label-free functional proteomics. Mol Cell Proteomics 6:1560-1573

36. Cutillas PR, Geering B, Waterfield MD et al (2005) Quantification of gel-separated proteins and their phosphorylation sites by LC-MS using unlabeled internal standards: analysis of phosphoprotein dynamics in a B cell lymphoma cell line. Mol Cell Proteomics 4:1038-1051

37. Bateman NW, Goulding SP, Shulman NJ et al (2014) Maximizing peptide identification events in proteomic workflows using datadependent acquisition (DDA). Mol Cell Proteomics 13:329-338

38. Alcolea MP, Casado P, Rodríguez-Prados J-C et al (2012) Phosphoproteomic analysis of leukemia cells under basal and drug-treated conditions identifies markers of kinase pathway activation and mechanisms of resistance. Mol Cell Proteomics 11:453-466

39. Cox J, Hein MY, Luber CA et al (2014) Accurate proteome-wide label-free quantification by delayed normalization and maximal peptide ratio extraction, termed MaxLFQ. Mol Cell Proteomics 13:2513-2526

40. Strittmatter EF, Ferguson PL, Tang K et al (2003) Proteome analyses using accurate mass and elution time peptide tags with capillary LC time-of-flight mass spectrometry. J Am Soc Mass Spectrom 14:980-991

41. Lange V, Picotti P, Domon B et al (2008) Selected reaction monitoring for quantitative proteomics: a tutorial. Mol Syst Biol 4:222

42. Gillet LC, Navarro P, Tate S et al (2012) Targeted data extraction of the MS/MS spectra generated by data-independent acquisition: a new concept for consistent and accurate proteome analysis. Mol Cell Proteomics 11:O111.016717

43. Parker BL, Yang G, Humphrey SJ et al (2015) Targeted phosphoproteomics of insulin signaling using data-independent acquisition mass spectrometry. Sci Signal 8:rs6

44. Sidoli S, Fujiwara R, Kulej K et al (2016) Differential quantification of isobaric phosphopeptides using data-independent acquisition mass spectrometry. Mol BioSyst 12 (8):2385-2388

45. Keller A, Bader SL, Kusebauch U et al (2016) Opening a SWATH window on posttranslational modifications: automated pursuit of modified peptides. Mol Cell Proteomics 15:1151-1163

46. Ong S-E, Blagoev B, Kratchmarova I et al (2002) Stable isotope labeling by amino acids in cell culture, SILAC, as a simple and accurate approach to expression proteomics. Mol Cell Proteomics 1:376-386

47. Zanivan S, Meves A, Behrendt K et al (2013) In vivo SILAC-based proteomics reveals phosphoproteome changes during mouse skin carcinogenesis. Cell Rep 3:552-566 
48. Shenoy A, Geiger T (2015) Super-SILAC: current trends and future perspectives. Expert Rev Proteomics 12:13-19

49. Thompson A, Schäfer J, Kuhn K et al (2003) Tandem mass tags: a novel quantification strategy for comparative analysis of complex protein mixtures by MS/MS. Anal Chem 75:1895-1904

50. Ross PL, Huang YN, Marchese JN et al (2004) Multiplexed protein quantitation in Saccharomyces cerevisiae using aminereactive isobaric tagging reagents. Mol Cell Proteomics 3:1154-1169

51. Li Z, Adams RM, Chourey K et al (2012) Systematic comparison of label-free, metabolic labeling, and isobaric chemical labeling for quantitative proteomics on LTQ Orbitrap Velos. J Proteome Res 11:1582-1590

52. Chelius D, Bondarenko PV (2002) Quantitative profiling of proteins in complex mixtures using liquid chromatography and mass spectrometry. J Proteome Res 1:317-323

53. Neilson KA, Ali NA, Muralidharan $S$ et al (2011) Less label, more free: approaches in label-free quantitative mass spectrometry. Proteomics 11:535-553

54. Perkins DN, Pappin DJ, Creasy DM et al (1999) Probability-based protein identification by searching sequence databases using mass spectrometry data. Electrophoresis 20:3551-3567

55. Clauser KR, Baker P, Burlingame AL (1999) Role of accurate mass measurement $(+/-10$ $\mathrm{ppm}$ ) in protein identification strategies employing MS or MS/MS and database searching. Anal Chem 71:2871-2882

56. MacCoss MJ, Wu CC, Yates JR (2002) Probability-based validation of protein identifications using a modified SEQUEST algorithm. Anal Chem 74:5593-5599

57. Cox J, Neuhauser N, Michalski A et al (2011) Andromeda: a peptide search engine integrated into the MaxQuant environment. J Proteome Res 10:1794-1805

58. Beausoleil SA, Villén J, Gerber SA et al (2006) A probability-based approach for highthroughput protein phosphorylation analysis and site localization. Nat Biotechnol 24:1285-1292

59. Savitski MM, Lemeer $S$, Boesche $M$ et al (2011) Confident phosphorylation site localization using the Mascot Delta Score. Mol Cell Proteomics 10:Ml10.003830

60. Chalkley RJ, Clauser KR (2012) Modification site localization scoring: strategies and performance. Mol Cell Proteomics 11:3-14
61. Baker PR, Trinidad JC, Chalkley RJ (2011) Modification site localization scoring integrated into a search engine. Mol Cell Proteomics 10:Ml11.008078

62. Lemeer S, Heck AJR (2009) The phosphoproteomics data explosion. Curr Opin Chem Biol 13:414-420

63. Sharma K, D'Souza RCJ, Tyanova $S$ et al (2014) Ultradeep human phosphoproteome reveals a distinct regulatory nature of Tyr and Ser/Thr-based signaling. Cell Rep 8:1583-1594

64. Olsen JV, Blagoev B, Gnad F et al (2006) Global, in vivo, and site-specific phosphorylation dynamics in signaling networks. Cell 127:635-648

65. Olsen JV, Vermeulen M, Santamaria A et al (2010) Quantitative phosphoproteomics reveals widespread full phosphorylation site occupancy during mitosis. Sci Signal 3:ra3

66. Landry CR, Levy ED, Michnick SW (2009) Weak functional constraints on phosphoproteomes. Trends Genet 25:193-197

67. Beltrao P, Trinidad JC, Fiedler D et al (2009) Evolution of phosphoregulation: comparison of phosphorylation patterns across yeast species. PLoS Biol 7:e1000134

68. Beltrao P, Bork P, Krogan NJ et al (2013) Evolution and functional cross-talk of protein post-translational modifications. Mol Syst Biol 9:714

69. Newman RH, Zhang J, Zhu H (2014) Toward a systems-level view of dynamic phosphorylation networks. Front Genet 5:263

70. Glickman JF (2012) Assay development for protein kinase enzymes. Eli Lilly \& Company and the National Center for Advancing Translational Sciences, Bethesda, MD. http:// www.ncbi.nlm.nih.gov/books/NBK91991/

71. Perfetto L, Briganti L, Calderone A et al (2016) SIGNOR: a database of causal relationships between biological entities. Nucleic Acids Res 44:D548-D554

72. Gnad F, Gunawardena J, Mann M (2011) PHOSIDA 2011: the posttranslational modification database. Nucleic Acids Res 39: D253-D260

73. Hu J, Rho H-S, Newman RH et al (2014) PhosphoNetworks: a database for human phosphorylation networks. Bioinformatics (Oxford, England) 30:141-142

74. Sadowski I, Breitkreutz B-J, Stark C et al (2013) The PhosphoGRID Saccharomyces cerevisiae protein phosphorylation site database: version 2.0 update. Database 2013: bat026 
75. Duan G, Li X, Köhn M (2015) The human DEPhOsphorylation database DEPOD: a 2015 update. Nucleic Acids Res 43: D531-D535

76. Zhang H, Zha X, Tan Y et al (2002) Phosphoprotein analysis using antibodies broadly reactive against phosphorylated motifs. J Biol Chem 277:39379-39387

77. Obenauer JC, Cantley LC, Yaffe MB (2003) Scansite 2.0: proteome-wide prediction of cell signaling interactions using short sequence motifs. Nucleic Acids Res 31:3635-3641

78. C. Chen and B.E. Turk (2010) Analysis of serine-threonine kinase specificity using arrayed positional scanning peptide libraries., Curr Protoc Mol Biol Chapter 18:Unit 18.14

79. Sidhu SS, Koide S (2007) Phage display for engineering and analyzing protein interaction interfaces. Curr Opin Struct Biol 17:481-487

80. Miller ML, Jensen LJ, Diella F et al (2008) Linear motif atlas for phosphorylationdependent signaling. Sci Signal 1:ra2

81. Hjerrild M, Stensballe A, Rasmussen TE et al (2004) Identification of phosphorylation sites in protein kinase A substrates using artificial neural networks and mass spectrometry. J Proteome Res 3:426-433

82. Linding R, Jensen LJ, Pasculescu A et al (2008) NetworKIN: a resource for exploring cellular phosphorylation networks. Nucleic Acids Res 36:D695-D699

83. Szklarczyk D, Franceschini A, Wyder S et al (2015) STRING vl0: protein-protein interaction networks, integrated over the tree of life. Nucleic Acids Res 43:D447-D452

84. Wagih O, Sugiyama N, Ishihama $\mathrm{Y}$ et al (2016) Uncovering phosphorylation-based specificities through functional interaction networks. Mol Cell Proteomics 15:236-245

85. Linding R, Jensen LJ, Ostheimer GJ et al (2007) Systematic discovery of in vivo phosphorylation networks. Cell 129:1415-1426

86. Subramanian A, Tamayo P, Mootha VK et al (2005) Gene set enrichment analysis: a knowledge-based approach for interpreting genome-wide expression profiles. Proc Natl Acad Sci U S A 102:15545-15550

87. Schacht T, Oswald M, Eils R et al (2014) Estimating the activity of transcription factors by the effect on their target genes. Bioinformatics (Oxford, England) 30:i401-i407

88. Drake JM, Graham NA, Stoyanova T et al (2012) Oncogene-specific activation of tyrosine kinase networks during prostate cancer progression. Proc Natl Acad Sci 109:1643-1648
89. Chen EY, Tan CM, Kou Y et al (2013) Enrichr: interactive and collaborative HTML5 gene list enrichment analysis tool. BMC Bioinformatics 14:128

90. Kuleshov MV, Jones MR, Rouillard AD et al (2016) Enrichr: a comprehensive gene set enrichment analysis web server 2016 update. Nucleic Acids Res 44(W1):W90-W97

91. Lachmann A, Ma'ayan A (2009) KEA: kinase enrichment analysis. Bioinformatics (Oxford, England) 25:684-686

92. Keshava Prasad TS, Goel R, Kandasamy K et al (2009) Human Protein Reference Database2009 update. Nucleic Acids Res 37: D767-D772

93. Huttlin EL, Jedrychowski MP, Elias JE et al (2010) A tissue-specific atlas of mouse protein phosphorylation and expression. Cell 143:1174-1189

94. de Graaf EL, Giansanti P, Altelaar AFM et al (2014) Single-step enrichment by Ti4+IMAC and label-free quantitation enables in-depth monitoring of phosphorylation dynamics with high reproducibility and temporal resolution. Mol Cell Proteomics 13:2426-2434

95. Wilm M, Mann M (1996) Analytical properties of the nanoelectrospray ion source. Anal Chem 68:1-8

96. Wilkes EH, Terfve C, Gribben JG et al (2015) Empirical inference of circuitry and plasticity in a kinase signaling network. Proc Natl Acad Sci U S A 112:7719-7724

97. Türei D, Korcsmáros T, Saez-Rodriguez J (2016) OmniPath: guidelines and gateway for literature-curated signaling pathway resources. Nat Methods 13:966-967

98. Benjamini Y, Hochberg Y (2000) On the adaptive control of the false discovery rate in multiple testing with independent statistics. J Educ Behav Stat 25:60-83

99. Mckinney W (2010) Data structures for statistical computing in python. Proceedings of the 9th python in science conference

100. Van Der Walt S, Colbert SC, Varoquaux G (2011) The NumPy Array: A Structure for Efficient Numerical Computation, Comput Sci Eng 13:22-30. https://doi.org/10. 1109/MCSE.2011.37

101. Kim S-Y, Volsky DJ (2005) PAGE: parametric analysis of gene set enrichment. BMC Bioinformatics 6:144

102. Jones E, Oliphant TE, Peterson P (2007) Python for scientific computing. Comput Sci Eng 9:10-20

103. Imamura $H$, Sugiyama $N$, Wakabayashi $M$ et al (2014) Large-scale identification of 
phosphorylation sites for profiling protein kinase selectivity. J Proteome Res 13:3410-3419

104. Newman RH, Hu J, Rho H-S et al (2013) Construction of human activity-based phosphorylation networks. Mol Syst Biol 9:655

105. Creixell P, Palmeri A, Miller CJ et al (2015) Unmasking determinants of specificity in the human kinome. Cell 163:187-201

106. Terfve CDA, Wilkes EH, Casado $\mathrm{P}$ et al (2015) Large-scale models of signal propagation in human cells derived from discovery phosphoproteomic data. Nat Commun 6:8033

107. Creixell P, Schoof EM, Simpson CD et al (2015) Kinome-wide decoding of networkattacking mutations rewiring cancer signaling. Cell 163:202-217

108. Hernandez-Armenta C, Ochoa D, Goncalves E et al (2016) Benchmarking substrate-based kinase activity inference using phosphoproteomic data. Bioinformatics 33 (12):1845-1851

109. Türei D, Földvári-Nagy L, Fazekas D et al (2015) Autophagy Regulatory Network - a systems-level bioinformatics resource for studying the mechanism and regulation of autophagy. Autophagy 11:155-165

110. Ma'ayan A, Jenkins SL, Neves S et al (2005) Formation of regulatory patterns during signal propagation in a Mammalian cellular network. Science (New York, NY) 309:1078-1083

111. Huang K-Y, Su M-G, Kao H-J et al (2016) dbPTM 2016: 10-year anniversary of a resource for post-translational modification of proteins. Nucleic Acids Res 44: D435-D446

112. Wagih O, Reimand J, Bader GD (2015) MIMP: predicting the impact of mutations on kinase-substrate phosphorylation. Nat Methods 12:531-533

113. Raza S, McDerment N, Lacaze PA et al (2010) Construction of a large scale integrated map of macrophage pathogen recognition and effector systems. BMC Syst Biol $4: 63$

114. Türei D, Papp D, Fazekas D et al (2013) NRF2-ome: an integrated web resource to discover protein interaction and regulatory networks of NRF2. Oxidative Med Cell Longev 2013:737591

115. Paz A, Brownstein Z, Ber Y et al (2011) SPIKE: a database of highly curated human signaling pathways. Nucleic Acids Res 39: D793-D799

116. Fazekas D, Koltai M, Türei D et al (2013) SignaLink 2 - a signaling pathway resource with multi-layered regulatory networks. BMC Syst Biol 7:7

117. Chun JN, Lim JM, Kang Y et al (2014) A network perspective on unraveling the role of TRP channels in biology and disease. Pflugers Arch 466:173-182

118. Cokelaer T, Pultz D, Harder LM et al (2013) BioServices: a common Python package to access biological Web Services programmatically. Bioinformatics 29:3241-3242

Open Access This chapter is licensed under the terms of the Creative Commons Attribution 4.0 International License (http://creativecommons.org/licenses/by/4.0/), which permits use, sharing, adaptation, distribution and reproduction in any medium or format, as long as you give appropriate credit to the original author(s) and the source, provide a link to the Creative Commons license and indicate if changes were made.

The images or other third party material in this chapter are included in the chapter's Creative Commons license, unless indicated otherwise in a credit line to the material. If material is not included in the chapter's Creative Commons license and your intended use is not permitted by statutory regulation or exceeds the permitted use, you will need to obtain permission directly from the copyright holder.

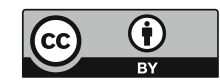

